\title{
Regulation of Synaptic Vesicle Budding and Dynamin Function by an EHD ATPase
}

\author{
Joel Jakobsson, ${ }^{1 *}$ Frauke Ackermann, ${ }^{1 *}$ Fredrik Andersson, ${ }^{1}$ Dan Larhammar, ${ }^{2}$ Peter Löw, ${ }^{1}$ and Lennart Brodin ${ }^{1}$ \\ ${ }^{1}$ Department of Neuroscience, Karolinska Institutet, S-171 77, Stockholm, Sweden, and ${ }^{2}$ Department of Neuroscience, Uppsala University, S-751 24, \\ Uppsala, Sweden
}

Eps15 homology domain-containing proteins (EHDs) are conserved ATPases implicated in membrane remodeling. Recently, EHD1 was found to be enriched at synaptic release sites, suggesting a possible involvement in the trafficking of synaptic vesicles. We have investigated the role of an EHD1/3 ortholog (l-EHD) in the lamprey giant reticulospinal synapse. 1-EHD was detected by immunogold at endocytic structures adjacent to release sites. In antibody microinjection experiments, perturbation of l-EHD inhibited synaptic vesicle endocytosis and caused accumulation of clathrin-coated pits with atypical, elongated necks. The necks were covered with helix-like material containing dynamin. To test whether l-EHD directly interferes with dynamin function, we used fluid-supported bilayers as in vitro assay. We found that l-EHD strongly inhibited vesicle budding induced by dynamin in the constant presence of GTP. l-EHD also inhibited dynamin-induced membrane tubulation in the presence of GTP $\gamma \mathrm{S}$, a phenomenon linked with dynamin helix assembly. Our in vivo results demonstrate the involvement of l-EHD in clathrin/dynamin-dependent synaptic vesicle budding. Based on our in vitro observations, we suggest that l-EHD acts to limit the formation of long, unproductive dynamin helices, thereby promoting vesicle budding.

\section{Introduction}

Three modes of synaptic vesicle endocytosis have been suggested to occur at nerve terminals: clathrin-mediated endocytosis, kiss-and-run recapture, and bulk endocytosis (Takei et al., 1996; Clayton et al., 2008; Dittman and Ryan, 2009; Granseth et al., 2009; Zhang et al., 2009; Hoopmann et al., 2010). However, the precise roles of these pathways remain unclear, in part due to lack of information about the molecular components used by each distinct pathway. One possible pathwayspecific protein is the intramembrane lipase Rolling blackout $(R b o)$. In a temperature-sensitive Drosophila Rbo mutant, clathrin-mediated endocytosis was found to proceed at the restrictive temperature, but bulk endocytosis was impaired (Vijayakrishnan et al., 2009). In vertebrates, syndapin/PACSIN has been implicated as a specific regulator of a form of bulk membrane retrieval activated during high rates of neurotransmitter release (Andersson et al., 2008; Clayton et al., 2009). Syndapin contains a membrane-bending F-BAR do-

\footnotetext{
Received March 11, 2011; revised Aug. 1, 2011; accepted Aug. 9, 2011.

Author contributions: J.J., F. Ackermann, P.L., and L.B. designed research; J.J., F. Ackermann, F. Andersson, D.L., P.L., and L.B. performed research; J.J., F. Ackermann, F. Andersson, D.L., P.L., and L.B. analyzed data; J.J., F. Ackermann, and L.B. wrote the paper.

This work was supported by The Swedish Research Council (21405 and DBRM), Hjärnfonden (L.B.), and a Postdoctoral Fellowship from The Deutsche Forschungsgemeinschaft (F. Ackermann). We thank T. C. Pucadyil and F. Salomons for advice and R. Lundmark, 0. Shupliakov, and J. Gregory for discussions and comments on the manuscript.

*J.J. and F. Ackermann contributed equally to this work.

The authors declare no competing financial interests.

Correspondence should be addressed to Lennart Brodin, Department of Neuroscience, Karolinska Institutet, S-17177, Stockholm, Sweden. E-mail: lennart.brodin@ki.se.

DOI:10.1523/JNEUROSCI.1289-11.2011

Copyright $\odot 2011$ the authors $\quad 0270-6474 / 11 / 3113972-09 \$ 15.00 / 0$
}

main, NPF motifs, and an SH3 domain that bind proteins implicated in actin regulation and membrane remodeling (Kessels and Qualmann, 2004; Braun et al., 2005; Itoh and De Camilli, 2006). Previous studies have documented critical roles of the $\mathrm{SH} 3$ interactions with $\mathrm{N}$-WASP and dynamin (Anggono et al., 2006; Andersson et al., 2008; Clayton et al., 2009). Less is known about the NPF motif-binding partners, which include Eps 15 homology domain-containing proteins (EHDs) (Braun et al., 2005). To learn more about the role of syndapin in synaptic vesicle recycling, we have begun to study these proteins.

The EHD family is comprised of four members in mammals and a single member in Drosophila and Caenorhabditis elegans (termed RME-1). EHD proteins contain an N-terminal G-domain linked via a helical region with a C-terminal EH domain. The G-domain exhibits structural similarity to the G-domain of dynamin but binds ATP instead of GTP (Daumke et al., 2007). The EH domain of EHDs preferentially binds proteins that contain acidic residues following the NPF tripeptide (Braun et al., 2005; Naslavsky and Caplan, 2011). Initial functional studies have implicated EHD as a regulator of the exit from the endocytic recycling compartment (Grant et al., 2001; Lin et al., 2001). Subsequently, EHD family members have been linked to multiple steps in endosomal trafficking pathways, as well as with other functions such as non-clathrin-mediated endocytosis of growth factor receptors and cell adhesion molecules (Naslavsky and Caplan, 2011). Recently, EHD1 was detected in nerve terminals (Wei et al., 2010), suggesting a possible involvement in synaptic vesicle trafficking. In mammals, EHD1, EHD3, and EHD4 are all expressed at significant levels in brain (Blume et al., 2007; Wei et al., 2010), which complicates studies using genetic approaches. To examine the possible involvement of EHDs in synaptic vesicle recy- 


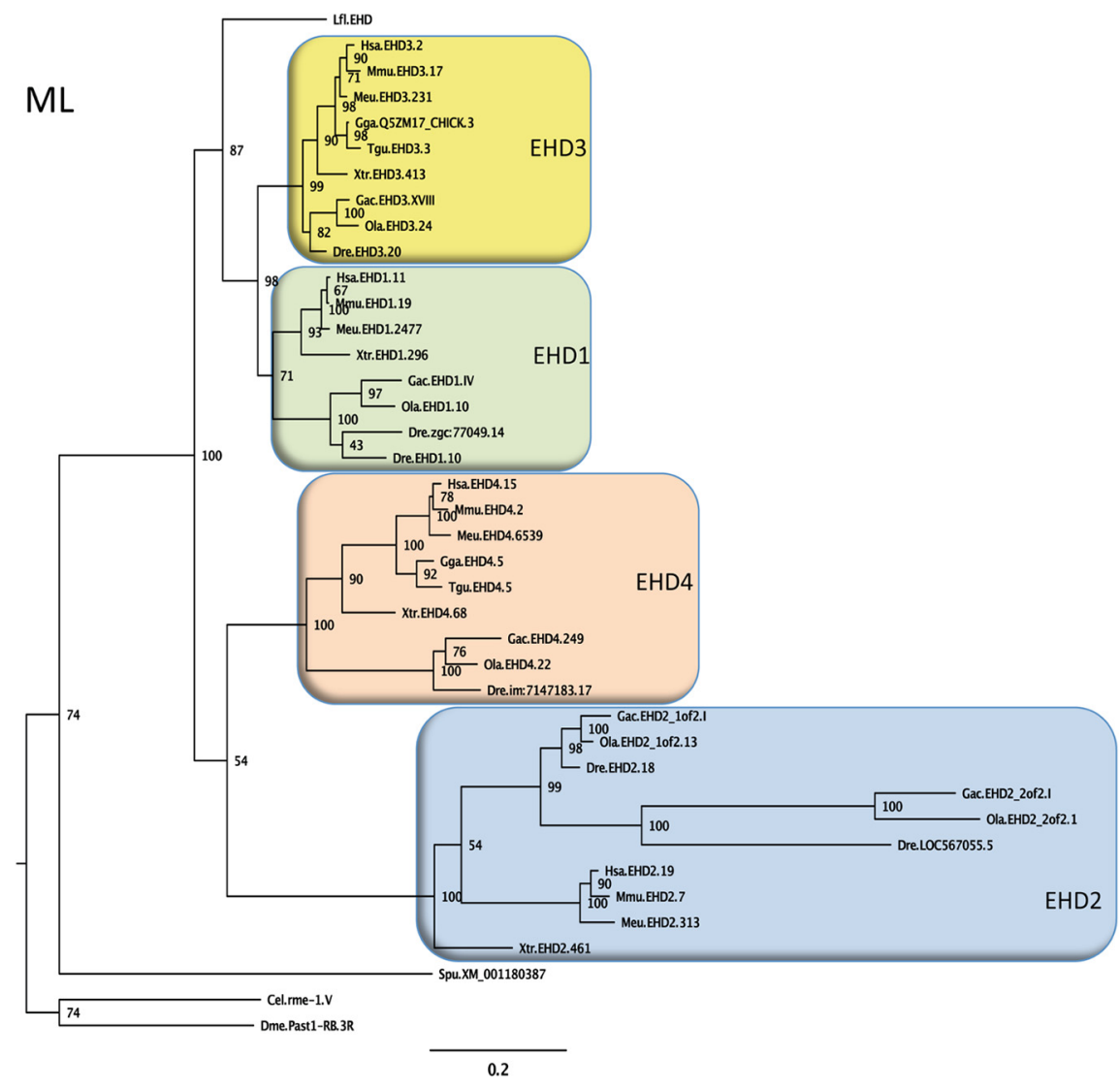

Figure 1. Phylogeny of EHD proteins. A phylogenetic quartet-puzzling maximum likelihood tree using protein sequences is shown. The first three letters of the sequence names are abbreviations of the species names, then follows EHD subtype (according to the ENSEMBL or NCBI databases), and number for the chromosome, group, or scaffold on which the gene is located. A few sequences have been assigned administrative names in the databases and these have been retained in the tree. Bootstrap values are shown at the nodes. Species abbreviations: Cel, Caenorhabditis elegans; Dme, Drosophila melanogaster; Dre, Danio rerio (zebrafish); Gac, Gasterosteus aculeatus (three-spined stickleback); Gga, Gallus gallus; Hsa, Homo sapiens; Meu, Macropus eugenii (Tammar wallaby); Mmu, Mus musculus; Lfl, Lampetra fluviatilis (=I-EHD); Ola, Oryzias latipes (medaka); Spu, Strongylocentrotus purpuratus (California purple sea urchin); Tgu, Taeniopygia guttata (zebra finch); Xtr, Xenopus tropicalis (Western clawed frog). The tree suggests that a single invertebrate EHD gene was quadrupled before the radiation of jawed vertebrates, but detailed analyses of the chromosomal locations of the EHD genes across these species will be required before this conclusion can be firmly drawn. Accession codes, the alignment, and the neighbor-joining tree can be obtained upon request.

cling, we have used the lamprey giant reticulospinal axon in which proteins can be perturbed locally at synaptic release sites.

\section{Materials and Methods}

Cloning of lamprey EHD. Total mRNA was isolated from lamprey (Lampetra fluviatilis of either sex) brains using a QuickPrep mRNA purification kit (GE Healthcare). Lamprey cDNA was constructed using an oligo(dT)-primer in a ThermoScript Reverse Transcriptase-PCR System (Invitrogen). Sequence data from the Petromyzon marinus genome-sequencing project was used for designing primers. Full-length lamprey EHD was cloned by PCR and the product was subcloned into a pCR2.1-TOPO vector (TOPO cloning kit, Invitrogen). The $\mathrm{EH}$ domains of lamprey intersectin and lamprey Eps15 were cloned with a similar approach. The Karolinska Institute Sequencing Core Facility (KISEQ) performed sequencing. Sequence alignment was performed using the ClustalW software (European Bioinformatics Institute).

Fusion proteins. Full-length lamprey EHD (1-EHD, amino acids 1-537), the EH domain of 1-EHD (amino acids 439-537), the EH domain of lamprey intersectin (amino acids 14-291), and the EH domain of lamprey Eps15 (amino acids 1-284) were PCR amplified and subcloned into pGEX-6P-2 vectors (GE Healthcare). The GST fusion proteins were expressed and purified on a glutathione coupled Hi-Trap column using standard methods (GE Healthcare). For immunization, GST was removed by using PreScission protease (GE Healthcare). The generations of GST-SH3 domains of lamprey endophilin and amphiphysin have been described previously (Shupliakov et al., 1997; Ringstad et al., 1999).

Antibodies. The EH domains of lamprey $\mathrm{EHD}$, intersectin, and Eps15 were used for immunization of rabbits. Each polyclonal antibody was affinity purified from serum on a $\mathrm{N}$-hydroxysuccinimide Hi-Trap column with the appropriate GST fusion protein covalently linked (GE Healthcare). Synaptotagmin antibodies were raised against the cytosolic region of rat synaptotagmin 1 (amino acids 96-421) and affinity purified from serum. These antibodies reacted with a $65 \mathrm{kDa}$ band in brain extracts from lamprey and rat (data not shown). The generation of antibodies to dynamin (LD-1) and VAMP2 have been described previously (Evergren et al., 2004a; Jakobsson et al., 2008). Control microinjections were performed with protein A-purified rabbit IgG (Sigma). Western blot of lamprey tissue extracts and rat brain extracts were performed using standard techniques (Jakobsson et al., 2008).

Immunoprecipitation. Lamprey brain tissue was homogenized in $20 \mathrm{~mm}$ HEPES, $150 \mathrm{~mm}$ $\mathrm{KCl}, 5 \mathrm{~mm} \mathrm{MgCl}_{2}$, and $10 \mathrm{~mm}$ EDTA, pH 7.40 (IP buffer), with protease inhibitors (protease inhibitor cocktail, Sigma-Aldrich). The homogenate was incubated at $4^{\circ} \mathrm{C}$ for $1 \mathrm{~h}$ with $1 \%$ Triton X-100 (Sigma Aldrich), and then centrifuged for $1 \mathrm{~h}$ at 45,000 rpm in a Ti-70 rotor (Beckman). The supernatant was preincubated for $1 \mathrm{~h}$ at $4^{\circ} \mathrm{C}$ with protein A Sepharose (GE Healthcare). Three micrograms of antibodies (EHD or control antibodies) were incubated with $5 \mu$ l of protein A Sepharose (GE Healthcare) in $0.2 \mathrm{ml}$ of IP buffer for $1 \mathrm{~h}$ at $4^{\circ} \mathrm{C}$ and then washed twice with $1 \mathrm{ml}$ of IP buffer (centrifugation for $5 \mathrm{~min}$ at $500 \times g$ ). Two hundred microliters of protein extract or IP buffer were added to the samples and incubated for $2 \mathrm{~h}$ at $4^{\circ} \mathrm{C}$. The bound material was isolated by centrifugation and washed two times with IP buffer $+1 \%$ Triton X-100 and two times with IP buffer. Bound proteins were eluted with $2 \times$ sample buffer. Bound and unbound material was analyzed by SDS-PAGE as well as Western blot using standard techniques.

Presynaptic microinjection. Microinjections were performed as previously described (Andersson et al., 2008; Jakobsson et al., 2008). Briefly, lampreys were anesthetized with MS-222 and the spinal cord was dissected and placed in a recording chamber with Ringer's solution maintained at $8^{\circ} \mathrm{C}$. Microinjections were monitored with a cooled CCD detector (Princeton Instruments). For localization studies, synaptotagmin antibodies (conjugated with Alexa 546, Invitrogen) were co-microinjected with EHD antibodies (conjugated with Alexa 488, Invitrogen). Images were obtained with a Nikon Eclipse C1 system using a $40 \times / 0.8$ NA objective (Nikon). In perturbation experiments, axons were injected with EHD antibodies and were then stimulated at $5 \mathrm{~Hz}$ (or, for control, maintained at rest in low- $\mathrm{Ca}^{2+}$ Ringer's solution) for $30 \mathrm{~min}$ and then fixed for $1 \mathrm{~h}$ in ice-cold $3 \%$ glutaraldehyde $/ 0.5 \%$ paraformaldehyde and $4 \%$ tannic acid in $0.1 \mathrm{M}$ cacodylic acid buffer, $\mathrm{pH} 7.40$. Spinal cords were then fixed overnight at $4{ }^{\circ} \mathrm{C}$ in $3 \%$ glutaraldehyde $/ 0.5 \%$ paraformaldehyde in $0.1 \mathrm{M}$ cacodylic acid buffer, $\mathrm{pH} 7.40$, followed by $1 \mathrm{~h}$ postfixation in $1 \%$ osmium tetroxide in $0.1 \mathrm{~m}$ cacodylate buffer. Fixed spinal cords were dehydrated in ethanol and embedded in Durcupan ACM. Serial ultrathin sections $(70 \mathrm{~nm})$ were cut with an ultratome and placed on formvar-coated grids, counterstained with uranyl acetate and 
lead citrate, and examined in a Tecnai 12 electron microscope (FEI). Attempts to microinject full-length 1-EHD failed due to clogging of the micropipette, presumably due to polymerization of the protein. Microinjection of the EH domain of 1-EHD followed by stimulation led to a massive loss of synaptic vesicles (control: $157.2 \pm 13.7$; EHD EH domain: $33.4 \pm$ 3.9 , mean \pm SEM, $n=5$ and 5 synapses), indicating a critical role of EH-NPF interactions. Further analysis of such interactions and identification of the participating proteins fall out of the scope of this study.

Combined microinjection-immunogold labeling experiments were performed as previously described (Evergren et al., 2004b). Axons were microinjected with EHD antibodies (Fig. 4) or GTP $\gamma \mathrm{S}$ or the endophilin $\mathrm{SH} 3$ domain (Fig. 5), stimulated at $5 \mathrm{~Hz}$ for $30 \mathrm{~min}$, and rapidly fixed in $3 \%$ paraformaldehyde and $0.1 \%$ glutaraldehyde in $0.1 \mathrm{M}$ phosphate buffer (4 h, pH 7.4). Longitudinal sections $(60 \mu \mathrm{m}$ thick) were cut from the ventral side of the spinal cords with a vibratome to make the interior of axons accessible to antibodies. Sections were labeled with dynamin antibodies or EHD antibodies followed by incubation with gold-conjugated secondary antibodies, and embedded and analyzed as described previously (Andersson et al., 2008).

Preparation of liposomes. The required lipids DOPC (0.79 mM), DOPS (0.15 mM), PIP ${ }_{2}$ (0.05 $\mathrm{mM})$, and, in the experiments with labeled membranes, RhodaminePE $(0.01 \mathrm{~mm})$ were mixed in a glass tube. Afterward the lipids were dried under a stream of argon gas and kept under vacuum overnight. Before use, lipids were hydrated in $500 \mu \mathrm{l}$ of $20 \mathrm{~mm}$ HEPES and $150 \mathrm{~mm} \mathrm{NaCl}, \mathrm{pH} 7.5$, for $30 \mathrm{~min}$ at $37^{\circ} \mathrm{C}$. Subsequently the solution was thawed and frozen three times. The lipids were extruded through a $100 \mathrm{~nm}$ filter forming unilamellar vesicles. The liposome stock solution ( $1 \mathrm{~mm}$ ) was also used for preparation of the SUPER-template assays and was stored in the dark at $4^{\circ} \mathrm{C}$ for up to 1 week. For sedimentation assay, purified 1-EHD protein was added at a concentration of $0.1 \mathrm{~mm}$ to liposomes. The protein-lipid solution was incubated for 60-90 min at room temperature. For sedimentation, the mixture was centrifuged in an airfuge for 60-90 min. The supernatant and pellet fractions were analyzed for the presence of 1-EHD with SDS-PAGE and Western blot using 1-EHD IgG. The amount of 1-EHD in each fraction was quantified by measuring the intensity with ImageJ. The sum of the intensity in the supernatant and pellet fractions was set to $100 \%$, and afterward, the percentage comprising the pellet fraction was calculated. For tubulation assay, a droplet of the protein-lipid solution was put on carboncoated copper mesh grids (200 mesh) and incubated $1 \mathrm{~min}$ before negative staining in $1 \%$ uranyl acetate. Tubular structures were analyzed in a Tecnai 12 electron microscope.

SUPER template assay. A $5 \mu \mathrm{l}$ silica bead suspension (Corpuscular) was incubated with 20 $\mu \mathrm{l}$ of liposome stock solution $(1 \mathrm{~mm})$ in a total volume of $100 \mu \mathrm{l}$ of $20 \mathrm{~mm}$ HEPES and $600 \mathrm{~mm}$
A

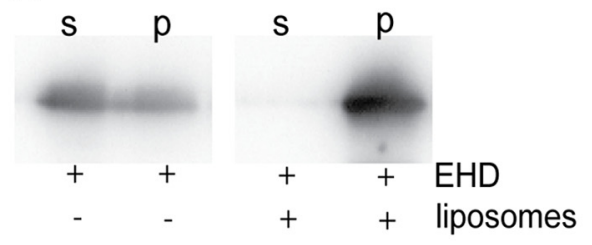

B
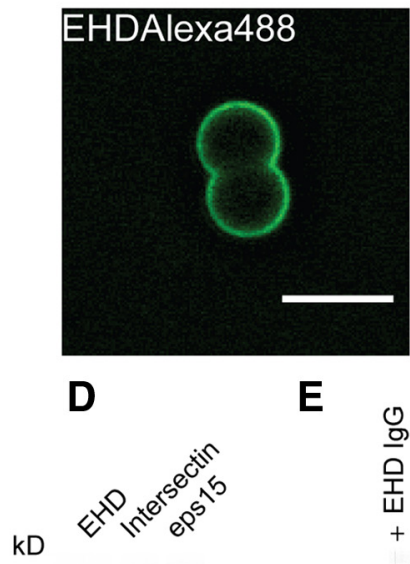

$\mathbf{E}$

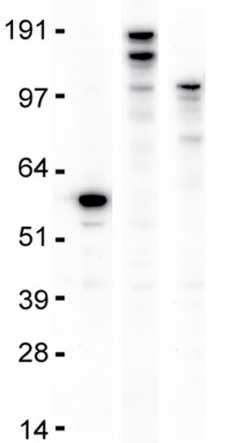

14 -

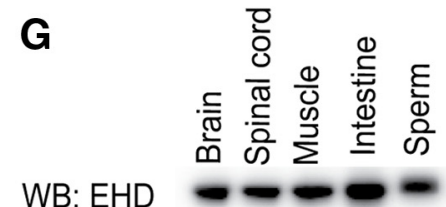

C
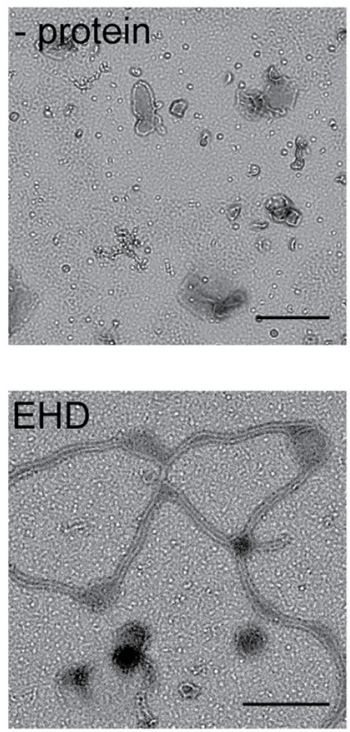

$\mathbf{F}$

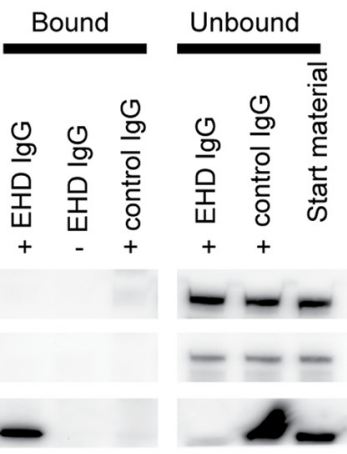

H
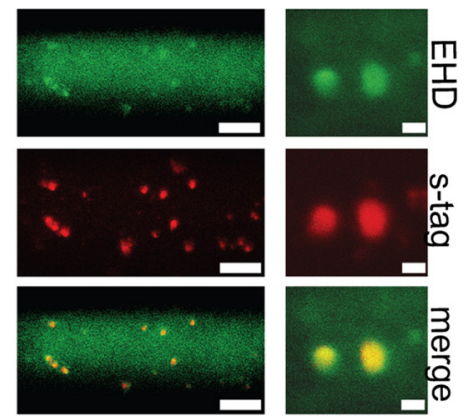

\section{WB: VAMP}

Figure 2. An EHD1/3 ortholog with conserved properties is enriched at synaptic release sites. $A$, Liposome sedimentation assay with I-EHD analyzed by Western blot. $\boldsymbol{B}$, Binding of Alexa 488 -labeled EHD $(0.5 \mu \mathrm{m})$ to unlabeled fluid-supported bilayers with excess membrane (SUPER templates). Scale bar, $5 \mu \mathrm{m}$. C, Tubulation of liposomes by I-EHD. Electron micrographs show negatively stained liposomes with no protein added (upper panel) or incubated with $1.6 \mu \mathrm{m}$ I-EHD (lower panel). Scale bars: $0.25 \mu \mathrm{m}$. D, Antibodies against the EH domain of I-EHD recognized a $60 \mathrm{kDa}$ protein in lamprey brain extract (left lane). No cross-reactivity with bands corresponding to intersectin (middle lane: Western blot with intersectin antibodies) or eps 15 (right lane: Western blot with eps 15 antibodies) was observed. $\boldsymbol{E}$, Immunoprecipitation of lamprey brain extract with I-EHD antibodies and control antibodies (Coomassie staining). $\boldsymbol{F}$, Western blot of bound and unbound material from immunoprecipitation with I-EHD antibodies. Neither intersectin nor eps 15 could be detected in the bound material. The I-EHD antibodies efficiently depleted I-EHD, but neither intersectin nor eps 15 , from the extract. G, Tissue distribution of I-EHD. Extracts from different lamprey tissues were blotted with I-EHD antibodies and with antibodies to VAMP2. H, I-EHD visualized at synaptic release sites in living reticulospinal axons following double microinjection of Alexa 488-labeled I-EHD antibodies (upper panels), and Alexa 546-labeled synaptotagmin antibodies (middle panels; lower panels show merged images). Low-magnification (left panels, scale bar, $30 \mu \mathrm{m}$ ) and high-magnification (right panels, scale bar, $2 \mu \mathrm{m}$ ) images are shown. Each spot corresponds to a synaptic vesicle cluster. The diffuse labeling in the axon may reflect unbound antibodies or antibodies bound to a cytoplasmic pool of EHD. The labeling pattern observed with the microinjected I-EHD antibodies is similar to that seen with antibodies to other endocytic proteins (Evergren et al., 2004a; Andersson et al., 2008; Jakobsson et al., 2008). 
$\mathrm{NaCl}, \mathrm{pH} 7.5$, for $30 \mathrm{~min}$ at room temperature in the dark. Subsequently $1 \mathrm{ml}$ of $\mathrm{H}_{2} \mathrm{O}$ was added to the beads to wash off excess unbound liposomes. Beads were spun down at $0.5 \mathrm{rpm}$ for $2 \mathrm{~min}$ and $1 \mathrm{ml}$ of the supernatant was removed. The washing step was repeated six times, and thereafter, the beads were resuspended in the remaining $100 \mu \mathrm{l}$ solution. Two microliters of SUPER templates were diluted in $100 \mu \mathrm{l}$ of $20 \mathrm{~mm}$ HEPES, $150 \mathrm{~mm} \mathrm{NaCl}, 1 \mathrm{~mm} \mathrm{MgCl}$, and $1 \mathrm{~mm}$ nucleotide buffer and imaged on glass bottom culture dishes (MatTek) coated with 2\% BSA. Protein stock solutions were added to the edge of the drop of buffer. The final protein concentrations were $0.5 \mu \mathrm{M}$. Fluorescence imaging was performed at room temperature on an inverted Angstrom Quorum Grid Confocal Microscope (Leica Microsystems) with a $63 \times$ (NA 1.4) oilimmersion objective. Alexa 488-labeled EHD was imaged with $490 \mathrm{~nm}$ excitation and $525 \mathrm{~nm}$ emission filters. For rhodamine-labeled membranes, $555 \mathrm{~nm}$ excitation and $605 \mathrm{~nm}$ emission filters were used. In Figure 6 , superimposed movies sampled at 1 image/ $2 \mathrm{~s}$ during $2 \min (A-C)$ and 5 $\min (D-F)$ are shown.

Purification of dynamin from rat brain. Forty grams of frozen rat brain was added to $150 \mathrm{ml}$ of ice-cold $20 \mathrm{~mm}$ Tris/ $\mathrm{HCl}, 1 \mathrm{~mm} \mathrm{CaCl}, 2 \mathrm{~mm}$ DTT, $0.1 \mathrm{mg} / \mathrm{ml} \mathrm{PMSF}$, and $2.5 \mathrm{mg} / \mathrm{ml}$ leupeptin buffer and homogenized with an Ultra Turrax. Subsequently, the homogenate was centrifuged at $15,000 \mathrm{rpm}$ for $30 \mathrm{~min}$ at $4^{\circ} \mathrm{C}$ in a Sorvall $5 \mathrm{~B}$ centrifuge (DuPont Instruments). The supernatant was discarded and the remaining pellet rehomogenized in $150 \mathrm{ml}$ of $20 \mathrm{~mm}$ Tris/ $\mathrm{HCl}, 0.1 \mathrm{~mm} \mathrm{CaCl}_{2}, 2 \mathrm{~mm}$ DTT, 0.1 $\mathrm{mg} / \mathrm{ml} \mathrm{PMSF}$, and $2.5 \mathrm{mg} / \mathrm{ml}$ leupeptin buffer, and centrifuged again for $30 \mathrm{~min}$ at $15,000 \mathrm{rpm}$ and $4^{\circ} \mathrm{C}$. After centrifugation, the resulting pellet was again rehomogenized in $150 \mathrm{ml}$ of $20 \mathrm{~mm}$ Tris/HCl, 2 mM EGTA, 2 mu EDTA, $250 \mathrm{~mm} \mathrm{NaCl}, 2 \mathrm{~mm}$ DTT, $0.1 \mathrm{mg} / \mathrm{ml} \mathrm{PMSF}$, and $2.5 \mathrm{mg} / \mathrm{ml}$ leupeptin buffer, stirred slowly for $30 \mathrm{~min}$ at $4^{\circ} \mathrm{C}$ and centrifuged for 30 min at $15,000 \mathrm{rpm}$ and $4^{\circ} \mathrm{C}$. The resultant supernatant was the dynamin extract, which was recentrifuged for $20 \mathrm{~min}$ at $15,000 \mathrm{rpm}$ and $4^{\circ} \mathrm{C}$ to remove trace particulate material. Ammonium sulfate was then added to $35 \%$ saturation to the extract and stirred for $30 \mathrm{~min}$ at $4^{\circ} \mathrm{C}$. Following centrifugation for $30 \mathrm{~min}$ at $15,000 \mathrm{rpm}$ and $4^{\circ} \mathrm{C}$, the pellet was resuspended in $15 \mathrm{ml}$ of $200 \mathrm{~mm} \mathrm{NaCl}, 20 \mathrm{~mm}$ HEPES, 1 mм DTT, $1 \mathrm{~mm}$ EDTA, and $0.1 \%$ Tween 80 buffer and dialyzed overnight at $4^{\circ} \mathrm{C}$ against $2000 \mathrm{ml}$ of the same buffer. The dialysate was centrifuged for $30 \mathrm{~min}$ at $50,000 \mathrm{rpm}$ at $4^{\circ} \mathrm{C}$ in an ultracentrifuge. The supernatant was filtered through a $0.2 \mu \mathrm{m}$ syringe filter (Pall Life Sciences). Concurrently, a GSTamphiphysin-SH3 or a GST-endophilin-SH3 column was equilibrated with the dialysis buffer. Subsequently, the filtered dialysate was loaded on the column at $4^{\circ} \mathrm{C}$. The column was washed with $200 \mathrm{~mm} \mathrm{NaCl}, 20 \mathrm{~mm}$ HEPES, and $1 \mathrm{~mm}$ DTT, pH 7.3. Dynamin was eluted with $6 \mathrm{ml}$ of $0.1 \mathrm{M}$ glycine, $\mathrm{pH} 2.8$. The protein solution was neutralized with $5 \mu \mathrm{l}$ of $1 \mathrm{M}$ Tris, $\mathrm{pH}$ 9.0. Fractions with the highest protein concentration were pooled and buffer was changed to $200 \mathrm{~mm} \mathrm{NaCl}, 20 \mathrm{~mm}$ HEPES, and 1 mM DTT, pH 7.4. Following concentration, the dynamin solution was stored in small aliquots at $-80^{\circ} \mathrm{C}$. Purity and activity of dynamin were analyzed by SDS-PAGE and Western blot as well as with the Supertemplate assay.

Fluorescence resonance energy transfer analysis. SUPER templates were diluted, $2 \mu \mathrm{l}$ in $100 \mu \mathrm{l}$ of $20 \mathrm{~mm}$ HEPES, $150 \mathrm{~mm} \mathrm{NaCl}, 1 \mathrm{~mm} \mathrm{MgCl}, 1 \mathrm{~mm}$ ATP, and $1 \mathrm{~mm}$ GTP buffer and imaged on glass bottom culture dishes (MatTek) coated with 2\% BSA. Labeled protein stock solutions (EHDAlexa 488; dynamin-Alexa 546; dynamin PH domain-Alexa 546; endophilin-Alexa 488; GST-Alexa 488) were added to the SUPER templates shortly before imaging. The final protein concentration was 0.2 $\mu \mathrm{M}$. Fluorescence resonance energy transfer (FRET) microscopy was performed using a Quorum Angstrom Grid Confocal Microscope (Leica Microsystems) equipped with separate filter wheels for excitation and emission. The following filter combinations were used: Alexa $488-$ excitation (ex.) 490/20 nm, emission (em.) 525/30 nm, Alexa 546-ex. 555/25 nm, em. 605/30 nm, FRET- ex. 490/20 nm, em. 605/30 nm. Images were acquired with a cooled CCD camera (ORCA AG, Hamamatsu) and analyzed using the ImageJ 1.43 image analysis software (Gordon et al., 1998). FRET efficiencies were calculated using the ImageJ PixFRET plug-in (Feige et al., 2005). Bleed-through intensities were measured in the FRET channel when only the donor or the acceptor protein was added to the SUPER templates. The intensities were cal-

\section{A I-EHD IgG microinjection}

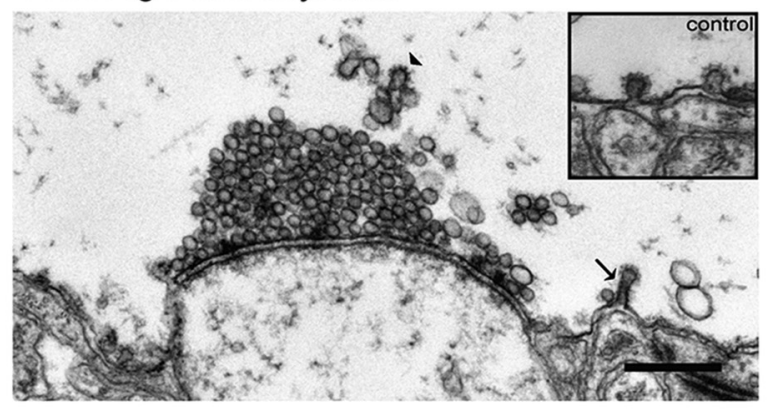

B
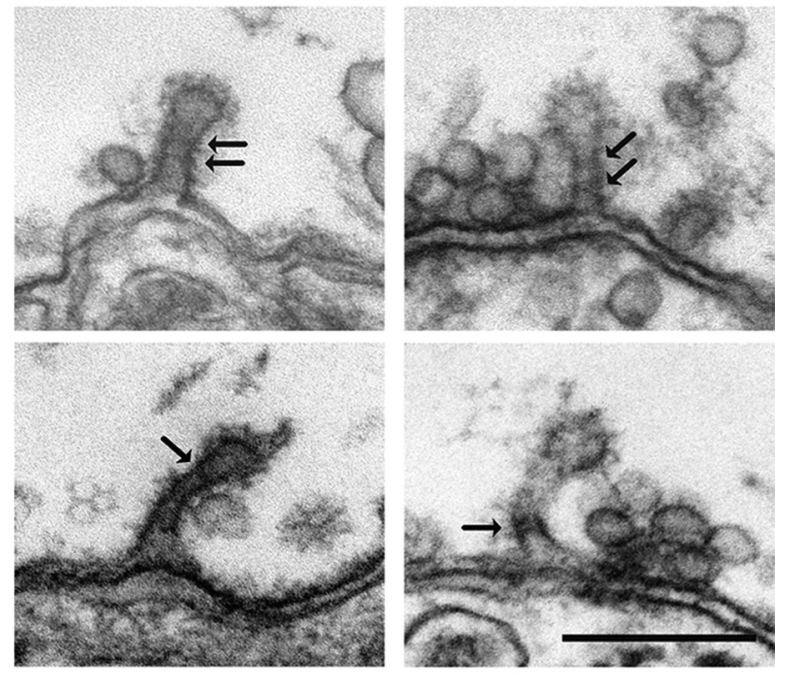

C

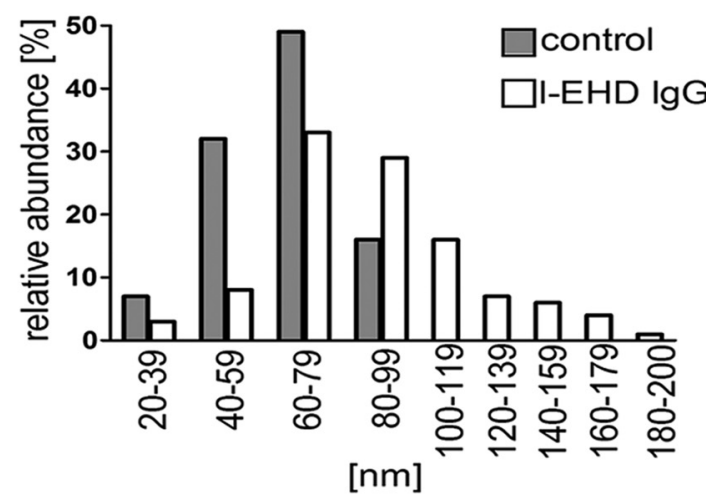

Figure 3. Perturbation of I-EHD at synaptic release sites causes accumulation of coated pits with elongated necks. $\boldsymbol{A}$, Electron micrograph of a synapse in a giant reticulospinal axon microinjected with I-EHD IgG followed by action potential stimulation at $5 \mathrm{~Hz}$ for 30 min. Note the clathrin-coated pit with an elongated neck (arrow; observed in 10 different antibody-injected giant axons in 3 different animals; coated pits in a control axons are shown in the inset). Clathrin coats visible on endosome-like structures above the synaptic vesicle cluster are indicated by an arrowhead. Scale bar, $0.5 \mu \mathrm{m}$. Axons microinjected with EHD antibodies, but maintained at rest in low-calcium Ringer, had a normal appearance (data not shown). $\boldsymbol{B}$, Examples of coated pits in the periactive zone of giant reticulospinal axons injected with I-EHD IgG followed by action potential stimulation. Helix-like structures at the elongated necks are indicated with arrows. Scale bar, $0.2 \mu \mathrm{m}$. C, Distribution of the length of clathrin-coated pits in I-EHD IgG-injected axons (open bars, $n=$ 117 ) and control axons (filled bars, $n=67$ ).

culated for each experiment separately. FRET efficiency values were calculated in three independent experiments from at least 60 beads to which acceptor as well as donor proteins had been added.

Statistics. Control synapses were collected in adjacent noninjected axons that received the same stimulation as the injected axons. The number of synaptic vesicles and coated pits were counted in center sections of 
serially cut synapses, normalized to $1 \mu \mathrm{m}$ active zone length (calculated using the ImageJ software from NIH), and, unless stated otherwise, presented as mean values \pm SEMs. Coated pit neck length was measured as the length from the base at the plasma membrane to the top of the coated pit membrane. Student's $t$ test or the MannWhitney test were used where appropriate.

\section{Results}

A conserved EHD1/3 ortholog is enriched at synaptic release sites

Our first aim was to determine the relationship between EHDs in the river lamprey (Lampetra fluviatilis, a commonly used model species) and those in other vertebrates. An EHD sequence with highest identity to human EHD1 (82\%) and EHD3 (83\%) was cloned from a river lamprey brain cDNA library. Searches within the sea lamprey (Petromyzon marinus) genome database did not provide evidence for the presence of other EHD genes, indicating that lampreys contain a single EHD gene. Figure 1 illustrates the position of river lamprey EHD (termed l-EHD below) in a phylogenetic tree. The l-EHD sequence clusters with high bootstrap support (87) with the tetrapod and teleost fish sequences for EHD1 and EHD3. As the l-EHD sequence branches off before the EHD1-EHD3 split, the tree suggests that l-EHD is an ortholog of the common ancestor of EHD1 and EHD3. The pattern of sequence relationships with four EHD orthologs found both in tetrapods and teleosts (and a few more recent duplicates in the latter) may suggest that a single invertebrate EHD gene was quadrupled in the two basal vertebrate tetraploidizations before the radiation of jawed vertebrates (Nakatani et al., 2007; Putnam et al., 2008). The basic properties of l-EHD were examined in vitro. Similar to EHDs studied previously (Daumke et al., 2007; Pant et al., 2009), recombinant l-EHD bound to liposomes (Fig. $2 A$; the pelleted fraction comprised $13.0 \pm 1.6 \%$ of the total amount in the absence of liposomes and $68.4 \pm 18.8$ in the presence of liposomes; $n=3, p<0.05$ ). Binding of l-EHD to lipid membranes was confirmed using SUPER templates (Fig. $2 B$, see also below). When added to liposomes, l-EHD converted them into narrow tubules (Fig. 2C).

Antibodies raised against the EH domain of 1-EHD reacted with a $60 \mathrm{kDa}$ band (Fig. $2 \mathrm{D}$ ) that was expressed in several tissues, including brain and spinal cord (Fig. 2G). The l-EHD antibodies did not cross-react with intersectin or Eps15, two major presynaptic EH domain proteins (Fig. 2D). As a further specificity test, immunoprecipitation experiments were performed. The l-EHD antibodies effectively precipitated l-EHD from brain extract without affecting the levels of intersectin or Eps15 (Fig. 2 E, F). To examine the localization of 1-EHD within giant reticulospinal axons, Alexa 488-labeled l-EHD antibodies and Alexa 546labeled synaptotagmin antibodies were co-microinjected. The labeling of the two antibodies colocalized (Fig. $2 H$ ), thus showing that l-EHD, like EHD1 (Wei et al., 2010), is enriched at synaptic release sites.

Perturbation of EHD causes accumulation of clathrin-coated pits with elongated dynamin-coated necks

The possible involvement of l-EHD in synaptic vesicle recycling was examined by using experimental conditions identical to
B

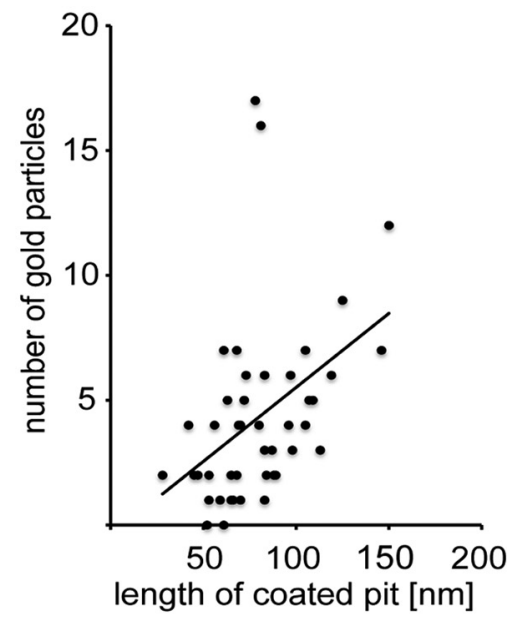

those used in our study of syndapin (Andersson et al., 2008). Following microinjection of l-EHD antibodies, axons were stimulated at $5 \mathrm{~Hz}$ for $30 \mathrm{~min}$ followed by rapid fixation. Analysis of serially sectioned synapses showed that the number of synaptic vesicles was reduced compared with stimulated control axons (Fig. 3 A; control 204.4 \pm 15.6; EHD IgG 67.24 $\pm 11.81, n=6$ and 6 synapses, $p<0.0001$ ), indicating that synaptic vesicle endocytosis was inhibited. Unlike the case of syndapin, however, no significant accumulation of membranous cisternae was observed (l-EHD antibodies, $10 \pm 2 \%$; control, $12 \pm 5 \%$; mean $\pm \mathrm{SD} ; n=$ 6 and 5 synapses). A further difference was that the number of coated pits at the plasma membrane in the periactive zone was significantly elevated by l-EHD antibodies (control $2.06 \pm 0.34$; EHD IgG $6.02 \pm 0.56, n=6$ and 6 synapses, $p<0.0005)$. These somewhat unexpected findings indicate that 1-EHD, rather than participating in a bulk membrane retrieval pathway, takes part in clathrin-mediated synaptic vesicle endocytosis at the plasma membrane.

The most notable finding from the EM analysis, however, was that many of the coated pits in 1-EHD antibody-injected axons had elongated, tubular necks (Fig. $3 A-C$; mean length in $\mathrm{nm} \pm$ SEM; control $64 \pm 19$; l-EHD IgG $91 \pm 2.9 ; n=67$ and 117 coated pits, $p<0.0001)$. The necks were surrounded by helix-like structures (Fig. $3 B$, arrows), an uncommon feature of coated pits under control conditions (Gad et al., 1998). Such helices may contain dynamin (Takei et al., 1995). This possibility was tested by immunogold labeling of axons cut open after microinjection of 1-EHD antibodies (Evergren et al., 2004b). We found that dynamin was distinctly accumulated along the necks (Fig. 4A). The level of dynamin per coated pit correlated positively with the length of coated pits (Fig. 4B). Quantitative analysis showed that $98 \%$ (150 of 153) of the coated pits with elongated necks (coated pit length of $>60 \mathrm{~nm}$ ) were labeled. To further examine a possible partnership between 1-EHD and dynamin, another set of combined microinjection-immunogold labeling experiments were performed. In this case, GTP $\gamma \mathrm{S}$ and the SH3 domain of endophilin were used, as these compounds can trap elongated and/or 


\section{A}

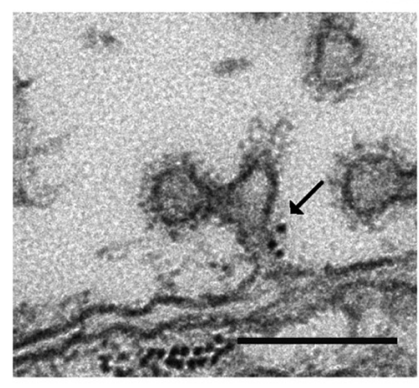

\section{GTPYS microinjection -}

\section{I-EHD immunogold labeling}
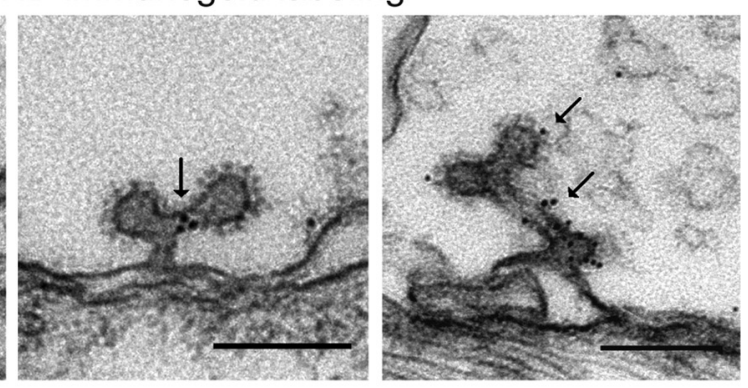

\section{B}

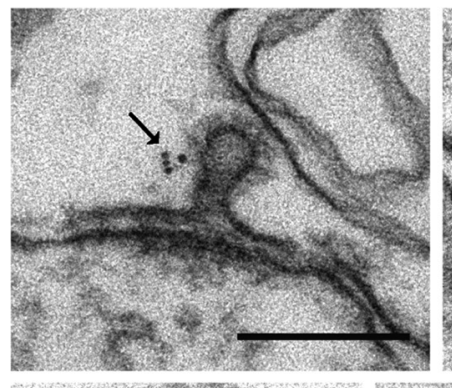

EndoSH3 microinjection I-EHD immunogold labeling
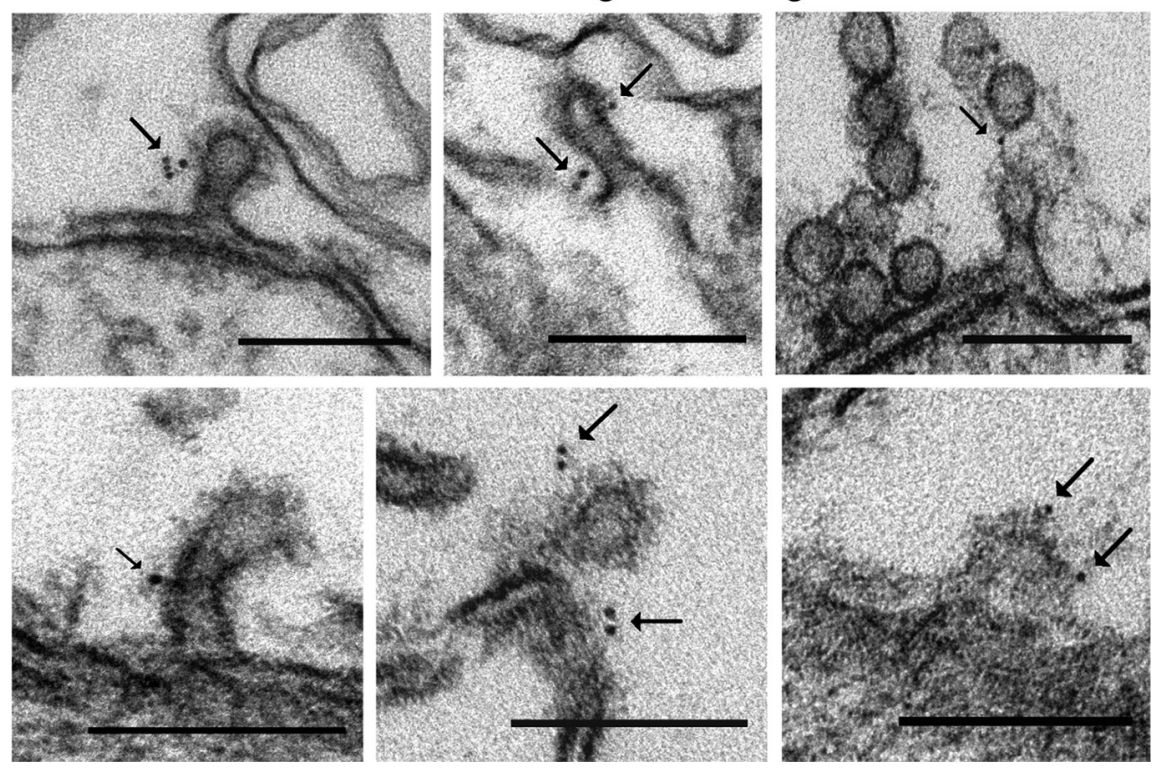

Figure 5. Immunogold localization of I-EHD at coated endocytic structures trapped after perturbation of dynamin function. $A$, Examples of the localization of I-EHD on coated structures in the periactive zone of axons microinjected with GTP $\gamma 5$ followed by action potential stimulation (Evergren et al., 2007). Gold particles are indicated by arrows. B, Examples of I-EHD localization on coated structures in the periactive zone of axons microinjected with the SH3 domain of endophilin followed by action potential stimulation (Gad et al., 2000). Scale bars, $0.15 \mu \mathrm{m}$.

branched dynamin-coated endocytic structures (Gad et al., 2000; Sundborger et al., 2011). In axons microinjected with either of these compounds, l-EHD immunolabeling could be detected at such endocytic structures (Fig. $5 A, B$ ). In both cases, $\sim 45 \%$ of the endocytic structures were labeled (214 of 484 in GTP $\gamma S$-injected axons; 79 of 175 in endoSH3-injected axons).

In addition to the effects of l-EHD antibodies on coated pits at the plasma membrane, we noted, in a few instances, cisternae with coated structures (Fig. 3A, arrowhead). However, as mentioned above, the number of cisternae in synaptic regions was not significantly elevated.

\section{EHD inhibits dynamin-induced vesiculation and tubulation in vitro}

The data presented above suggested that EHD may affect the function of dynamin. To test this possibility, we monitored dynamin function in real time using fluid-supported lipid bilayers with excess membrane (SUPER templates) (Pucadyil and Schmid, 2008). As shown in Figure $6 \mathrm{~A}$, addition of dynamin to rhodamine-labeled SUPER templates in the constant presence of
GTP induced a massive burst of vesiculation (mean number of vesicles/bead $26.7 \pm 8.1, n=3$ independent experiments). When dynamin was coapplied with EHD, however, vesiculation was strongly suppressed (Fig. $6 B ; 5.60 \pm 0.99$, $n=3, p<0.05)$. A similar inhibition was seen when the SUPER templates were preincubated with EHD before addition of dynamin. EHD alone did not induce vesiculation (data not shown).

In view of recent models of dynamin function (Bashkirov et al., 2008; Pucadyil and Schmid, 2008; see also Discussion), it seemed plausible that the effect of EHD could be due either to inhibition of the assembly of dynamin into helices or to inhibition of their disassembly. Our in vivo observations seemed to favor the former possibility. We addressed this question by modifying the experimental conditions. Long, dynamin-coated tubules can form in vitro in the presence of GTP $\gamma S$ due to the fact that assembly but not disassembly can occur under such conditions (Takei et al., 1995; Zhang and Hinshaw, 2001; Bashkirov et al., 2008). Therefore, we added dynamin to SUPER templates in the constant presence of GTP $\gamma \mathrm{S}$. In this case, instead of vesicles, tubular extensions formed from the SUPER template surface (Fig. $6 D ; 22.43 \pm 1.62 \%$ beads with tubules, mean of three experiments). Such tubule formation was strongly suppressed when dynamin was coapplied with EHD (Fig. $6 E, 7.03 \pm 0.72 \% n=42$, $p<0.0001$ ), indicating that l-EHD indeed inhibits dynamin assembly. Control experiments were performed in which ATP was replaced with ATP $\gamma$ S. The inhibitory effect of l-EHD on dynamin-induced vesiculation (Fig. 6C, $12.63 \pm 2.69, n=3$, $p<0.05$ ) as well as tubulation (Fig. $6 F, 16.61 \pm 1.28 \%, n=3, p<$ $0.0001)$ was significantly reduced under these conditions. The effect of l-EHD on dynamin function was thus not due to an unspecific increase in the protein concentration.

The observations described above suggested that EHD and dynamin can interact physically. We first examined this possibility with GST pulldown assays using lamprey spinal cord extract, with varying binding conditions (different detergents, salts, and nucleotides). Neither full-length GST-1-EHD nor the GSTcoupled EH domain of l-EHD bound dynamin at detectable levels (data not shown). However, interactions between synaptic proteins may in some cases require a lipid environment to become evident (Guan et al., 2008). This possibility was examined in FRET experiments using the SUPER templates and different combinations of labeled proteins (Fig. 7A-E). When Alexa 488labeled 1-EHD was applied together with Alexa 546-labeled fulllength dynamin, a FRET signal was recorded (Fig. 7B). Its magnitude was comparable to that induced by applying full-length dynamin together with its established binding partner endophilin (Fig. 7D). The l-EHD-full-length dynamin signal was significantly higher than the signals detected with two negative control pairs of proteins: 
GST-full-length dynamin (Fig. 7A) and 1EHD-PH domain of dynamin (Fig. 7C). We conclude that EHD and dynamin can come in close proximity to each other at lipid membranes $(<10 \mathrm{~nm}$; Taraska and Zagotta, 2010).

\section{Discussion}

At the onset of this study, we considered the possibility that EHD might participate in a syndapin-dependent bulk membrane retrieval pathway (Andersson et al., 2008; Clayton et al., 2009). Our findings do not, however, support this hypothesis. Perturbation of syndapin results in massive accumulation of large cisternae around release sites, but does not affect clathrincoated pits at the plasma membrane (Andersson et al., 2008). In contrast, we did not detect any accumulation of membranous cisternae after perturbation of EHD, while coated pits with an atypical morphology accumulated at the plasma membrane. These observations, along with the occurrence of l-EHD at coated endocytic intermediates, points to a role of 1-EHD in clathrin- and dynaminmediated synaptic vesicle endocytosis. The fact that we detected l-EHD labeling only on part of the endocytic structures examined $(45 \%)$ may potentially be explained by the existence of two parallel pathways: one EHD-independent but dynamin-dependent pathway and one dependent on both proteins. However, we favor the possibility of a single pathway, and that the incomplete labeling reflected a low l-EHD immunogold signal. Future studies with more sensitive EHD antibodies will hopefully answer this question. Whether or not the interaction between syndapin and EHD has functional significance in nerve terminals is presently not clear. We note that many other proteins interact with EHDs (Naslavsky and Caplan, 2011).

The clathrin-mediated pathway is the most well defined mechanism of synaptic vesicle recycling (Dittman and Ryan, 2009). A clathrin-coated membrane patch grows and invaginates in a process involving several accessory factors. Following formation of a deeply invaginated coated pit, the neck is surrounded by a dynamin helix, which plays a critical role in membrane fission. Based on initial in vitro studies of dynamin, it was proposed that assembled dynamin helices undergo a conformational change upon GTP hydrolysis (i.e., upon addition of GTP), which causes tension in the membrane leading to fission (Stowell et al., 1999; Zhang and Hinshaw, 2001; Roux et al., 2006). More recent studies performed in the constant presence of GTP have led to a somewhat different view of dynamin's role in membrane fission (Roux and Antonny, 2008). Dynamin alone can drive vesiculation (as confirmed here) by accumulating at self-limited assemblies that continuously cycle in the membrane (Pucadyil and Schmid, 2008). When applied to lipid tubes, dynamin was found to assemble into short spirals, causing narrowing of the tubes. GTP hydrolysis-driven disassembly of dynamin spirals appeared to result in an unstable state promoting membrane fission (Bashkirov et al., 2008; see also Roux et al., 2006). In contrast, long dynamin
Tubulation
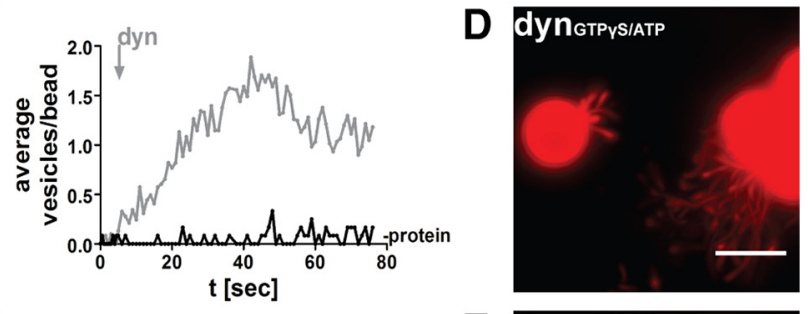

E dyn+EHDorTsSATP
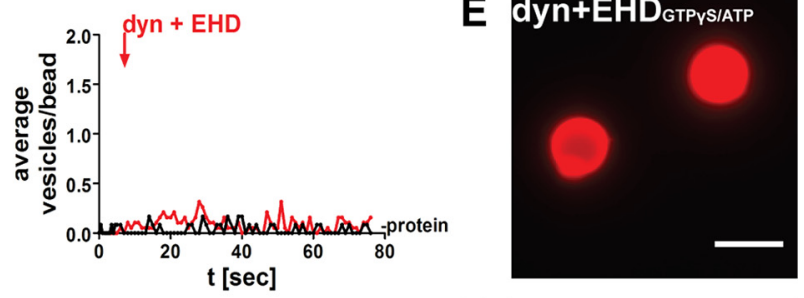

$\mathbf{F}$
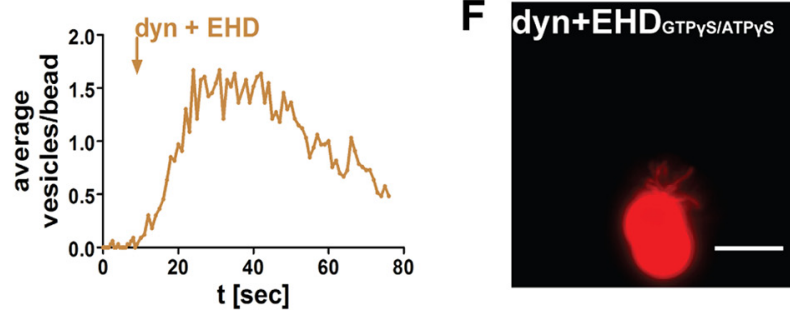

Figure 6. EHD modulates dynamin function in vitro. $\boldsymbol{A}$, Vesiculation from rhodamine-labeled SUPER templates induced by formation of narrow tubules (1 mm GTP $\gamma S$ and ATP present in $\boldsymbol{D}-\boldsymbol{F}$ ). $\boldsymbol{E}$, Tubule formation was suppressed when dynamin was coapplied with I-EHD. $\boldsymbol{F}$, Reduced inhibitory effect of I-EHD on dynamin-induced tubulation in the constant presence of GTP $\gamma S$ after replacement of ATP with ATP $\gamma$ S. Scale bars, $5 \mu \mathrm{m}$.

spirals formed in the absence of GTP were found to be ineffective in inducing fission upon addition of GTP. When considering dynamin function in cells, actions of accessory proteins that regulate dynamin function need to be taken into account. For instance, proteins containing a dynamin-binding SH3 domain and a BAR domain, such as endophilin and SNX9, can promote assembly of dynamin at membranes (Ramachandran and Schmid, 2008; Sundborger et al., 2011; see also Takei et al., 1999; Lackner et al., 2009). In accordance, perturbation of dynamin-SH3 domain interactions at synapses causes trapping of coated pits with short necks lacking dynamin helices (Shupliakov et al., 1997; Gad et al., 2000; Sundborger et al., 2011). Unexpectedly, we found the opposite effect after perturbation of EHD; coated pits with elongated necks surrounded by dynamincontaining material occurred at the plasma membrane. This observation suggests the existence of an in vivo mechanism that acts to limit excessive assembly of dynamin helices (Fig. $7 F$ ). It thereby implies that the self-limiting property of dynamin assemblies in vitro (Pucadyil and Schmid, 2008) is not sufficient to control helix length in vivo. Such an extrinsic limiting mechanism would be expected to act in balance with the assembly-stimulating factors discussed above, as it could otherwise prevent the nucleation of helices. It is possible that the EHD-dependent mechanism is regulated such that it is not activated until after a short helix has formed.

While we find it plausible that other proteins contribute to the presynaptic function of EHD, it is notable that the present in vitro tubulation, vesiculation, and FRET experiments indicate that EHD can interact directly with dynamin at membranes. How might such an interaction occur? The precise function of EHD 
A

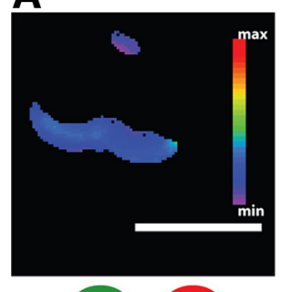

Gri $-D_{m i n}$

E

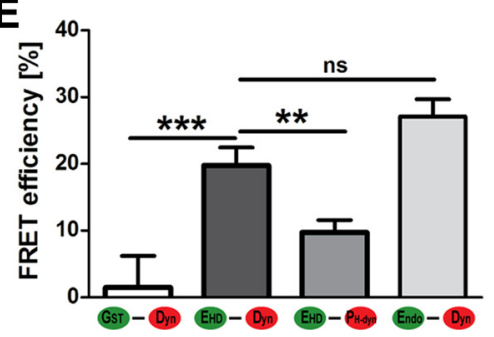

$E_{10}-D_{m i n}$
C
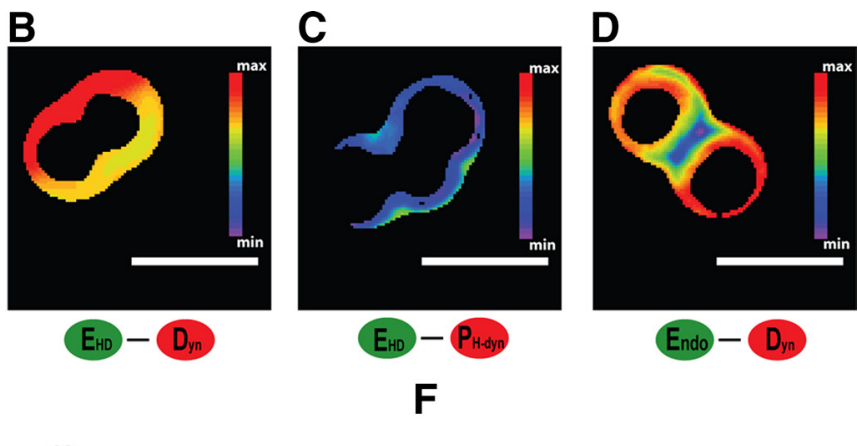

$\mathbf{F}$

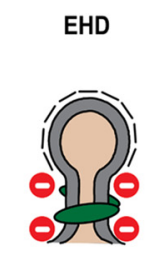

Figure 7. Association between EHD and dynamin at membranes studied with FRET. $\boldsymbol{A}-\boldsymbol{D}$, FRET efficiency between different Alexa-labeled proteins measured on the surface of SUPER templates. $A$, Alexa 488-labeled GST and Alexa 546-labeled full-length dynamin. B, Alexa 488-labeled I-EHD and Alexa 546-labeled full-length dynamin. C, Alexa 488-labeled I-EHD and Alexa 546labeled pleckstrin-homology (PH) domain of dynamin. D, Alexa 488-labeled full-length endophilin and Alexa 546-labeled fulllength dynamin. $\boldsymbol{E}$, Quantification of three independent FRET experiments for each combination of proteins (mean \pm SEM, $\left.{ }^{* * *} p<0.001,{ }^{* *} p<0.005\right)$. $\boldsymbol{F}$, EHD appears to counteract excessive assembly of dynamin helices. Such an effect may be expected to promote vesicle budding as recent studies of dynamin function indicate that short helices promote membrane fission more effectively than long helices.

proteins in relation to membrane remodeling is not yet fully clear, which leaves us to speculate. One possibility could be that EHD acts by sequestering dynamin at membranes, thereby counteracting growth of dynamin spirals. Preliminary data from experiments measuring the association of dynamin to membranes by FRET and FRAP are consistent with this possibility (F. Ackermann, L.B, unpublished observations). Another possibility is that EHD assembles into a ring above or below the dynamin spiral to sterically limit spiral growth. This explanation appears to be consistent with the observation that EHD2 can form rings (rather than helices) on lipid tubules with a diameter corresponding to that of the neck of a coated pit (Daumke et al., 2007). The inhibitory effect of l-EHD on dynamin-induced vesiculation and tubulation was found to be relieved when ATP was replaced with ATP $\gamma$ S, suggesting that cycles of ATP hydrolysis are involved. Further experiments will be required to address the mechanism behind this effect. We note that effects of nucleotides on the behavior of EHDs at membranes depend on the experimental conditions, including the EHD isoform used and the composition of lipids in the assay system (Daumke et al., 2007; Pant et al., 2009). Although EHDs exhibit similarities to dynamin with regard to structure and membrane-stimulated nucleotide hydrolysis, it is unclear to what extent the two proteins can be compared functionally. For instance, it is not yet clear whether EHD assemblies undergo structural changes upon ATP binding or hydrolysis, and the rate of membrane-stimulated ATP hydrolysis by EHD2 appears to be orders of magnitude slower than that of GTP hydrolysis by dynamin (Daumke et al., 2007).

In conclusion, we have found that a conserved, brainexpressed EHD1/3-type ATPase is enriched at synaptic release sites. It associates with endocytic structures and its perturbation impairs synaptic vesicle endocytosis, leaving coated pits with long, dynamin-coated necks at the plasma membrane. Under in vitro conditions EHD1/3 inhibits dynamin-dependent vesiculation and tubulation and interacts with dynamin at membranes

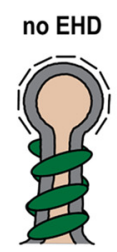

as indicated by FRET. We hypothesize that EHD $1 / 3$ acts by limiting the length of dynamin helices. As short helices are most effective in inducing membrane fission, such an influence on dynamin assembly would be expected to promote synaptic vesicle budding.

\section{References}

Andersson F, Jakobsson J, Löw P, Shupliakov O, Brodin L (2008) Perturbation of syndapin/ PACSIN impairs synaptic vesicle recycling evoked by intense stimulation. J Neurosci 28:3925-3933.

Anggono V, Smillie KJ, Graham ME, Valova VA, Cousin MA, Robinson PJ (2006) Syndapin I is the phosphorylation-regulated dynamin I partner in synaptic vesicle endocytosis. Nat Neurosci 9:752-760.

Bashkirov PV, Akimov SA, Evseev AI, Schmid SL, Zimmerberg J, Frolov VA (2008) GTPase cycle of dynamin is coupled to membrane squeeze and release, leading to spontaneous fission. Cell 135:1276-1286.

Blume JJ, Halbach A, Behrendt D, Paulsson M, Plomann M (2007) EHD proteins are associated with tubular and vesicular compartments and interact with specific phospholipids. Exp Cell Res 313:219-231.

Braun A, Pinyol R, Dahlhaus R, Koch D, Fonarev P, Grant BD, Kessels MM, Qualmann B (2005) EHD proteins associate with syndapin I and II and such interactions play a crucial role in endosomal recycling. Mol Biol Cell 16:3642-3658.

Clayton EL, Evans GJ, Cousin MA (2008) Bulk synaptic vesicle endocytosis is rapidly triggered during strong stimulation. J Neurosci 28:6627-6632.

Clayton EL, Anggono V, Smillie KJ, Chau N, Robinson PJ, Cousin MA (2009) The phospho-dependent dynamin-syndapin interaction triggers activity-dependent bulk endocytosis of synaptic vesicles. J Neurosci 29:7706-7717.

Daumke O, Lundmark R, Vallis Y, Martens S, Butler PJ, McMahon HT (2007) Architectural and mechanistic insights into an EHD ATPase involved in membrane remodelling. Nature 449:923-927.

Dittman J, Ryan TA (2009) Molecular circuitry of endocytosis at nerve terminals. Annu Rev Cell Dev Biol 25:133-160.

Evergren E, Marcucci M, Tomilin N, Löw P, Slepnev V, Andersson F, Gad H, Brodin L, De Camilli P, Shupliakov O (2004a) Amphiphysin is a component of clathrin coats formed during synaptic vesicle recycling at the lamprey giant synapse. Traffic 5:514-528.

Evergren E, Tomilin N, Vasylieva E, Sergeeva V, Bloom O, Gad H, Capani F, Shupliakov O (2004b) A pre-embedding immunogold approach for detection of synaptic endocytic proteins in situ. J Neurosci Methods 135:169-174.

Evergren E, Gad H, Walther K, Sundborger A, Tomilin N, Shupliakov O (2007) Intersectin is a negative regulator of dynamin recruitment to the synaptic endocytic zone in the central synapse. J Neurosci 27:379-390.

Feige JN, Sage D, Wahli W, Desvergne B, Gelman L (2005) PixFRET, an ImageJ plug-in for FRET calculation that can accommodate variations in spectral bleed-throughs. Microsc Res Tech 68:51-58.

Gad H, Löw P, Zotova E, Brodin L, Shupliakov O (1998) Dissociation between $\mathrm{Ca}^{2+}$-triggered synaptic vesicle exocytosis and clathrin-mediated endocytosis at a central synapse. Neuron 21:607-616.

Gad H, Ringstad N, Löw P, Kjaerulff O, Gustafsson J, Wenk M, Di Paolo G, Nemoto Y, Crum J, Ellisman MH, De Camilli P, Shupliakov O, Brodin L (2000) Fission and uncoating of synaptic clathrin-coated vesicles are perturbed by disruption of interactions with the $\mathrm{SH} 3$ domain of endophilin. Neuron 27:301-312.

Gordon GW, Berry G, Liang XH, Levine B, Herman B (1998) Quantitative fluorescence resonance energy transfer measurements using fluorescence microscopy. Biophys J 74:2702-2713.

Granseth B, Odermatt B, Royle SJ, Lagnado L (2009) Comment on "The 
dynamic control of kiss-and-run and vesicular reuse probed with single nanoparticles." Science 325:1499; author reply 1499.

Grant B, Zhang Y, Paupard MC, Lin SX, Hall DH, Hirsh D (2001) Evidence that RME-1, a conserved C. elegans EH-domain protein, functions in endocytic recycling. Nat Cell Biol 3:573-579.

Guan R, Dai H, Rizo J (2008) Binding of the Munc13-1 MUN domain to membrane-anchored SNARE complexes. Biochemistry 47:1474-1481.

Hoopmann P, Punge A, Barysch SV, Westphal V, Bückers J, Opazo F, Bethani I, Lauterbach MA, Hell SW, Rizzoli SO (2010) Endosomal sorting of readily releasable synaptic vesicles. Proc Natl Acad Sci U S A 107:19055-19060.

Itoh T, De Camilli P (2006) BAR, F-BAR (EFC) and ENTH/ANTH domains in the regulation of membrane-cytosol interfaces and membrane curvature. Biochim Biophys Acta 1761:897-912.

Jakobsson J, Gad H, Andersson F, Löw P, Shupliakov O, Brodin L (2008) Role of epsin 1 in synaptic vesicle endocytosis. Proc Natl Acad Sci U S A 105:6445-6450.

Kessels MM, Qualmann B (2004) The syndapin protein family: linking membrane trafficking with the cytoskeleton. J Cell Sci 117:3077-3086.

Lackner LL, Horner JS, Nunnari J (2009) Mechanistic analysis of a dynamin effector. Science 325:874-877.

Lin SX, Grant B, Hirsh D, Maxfield FR (2001) Rme-1 regulates the distribution and function of the endocytic recycling compartment in mammalian cells. Nat Cell Biol 3:567-572.

Nakatani Y, Takeda H, Kohara Y, Morishita S (2007) Reconstruction of the vertebrate ancestral genome reveals dynamic genome reorganization in early vertebrates. Genome Res 17:1254-1265.

Naslavsky N, Caplan S (2011) EHD proteins: key conductors of endocytic transport. Trends Cell Biol 21:122-131.

Pant S, Sharma M, Patel K, Caplan S, Carr CM, Grant BD (2009) AMPH-1/ Amphiphysin/Bin1 functions with RME-1/Ehd1 in endocytic recycling. Nat Cell Biol 11:1399-1410.

Pucadyil TJ, Schmid SL (2008) Real-time visualization of dynamincatalyzed membrane fission and vesicle release. Cell 135:1263-1275.

Putnam NH, Butts T, Ferrier DE, Furlong RF, Hellsten U, Kawashima T, Robinson-Rechavi M, Shoguchi E, Terry A, Yu JK, Benito-Gutiérrez EL, Dubchak I, Garcia-Fernàndez J, Gibson-Brown JJ, Grigoriev IV, Horton AC, de Jong PJ, Jurka J, Kapitonov VV, Kohara Y, et al. (2008) The amphioxus genome and the evolution of the chordate karyotype. Nature 453:1064-1071.

Ramachandran R, Schmid SL (2008) Real-time detection reveals that effectors couple dynamin's GTP-dependent conformational changes to the membrane. EMBO J 27:27-37.
Ringstad N, Gad H, Löw P, Di Paolo G, Brodin L, Shupliakov O, De Camilli P (1999) Endophilin/SH3p4 is required for the transition from early to late stages in clathrin-mediated synaptic vesicle endocytosis. Neuron 24:143-154.

Roux A, Antonny B (2008) The long and short of membrane fission. Cell 135:1163-1165.

Roux A, Uyhazi K, Frost A, De Camilli P (2006) GTP-dependent twisting of dynamin implicates constriction and tension in membrane fission. Nature 441:528-531.

Shupliakov O, Löw P, Grabs D, Gad H, Chen H, David C, Takei K, De Camilli P, Brodin L (1997) Synaptic vesicle endocytosis impaired by disruption of dynamin-SH3 domain interactions. Science 276:259-263.

Sundborger A, Soderblom C, Vorontsova O, Evergren E, Hinshaw JE, Shupliakov O (2011) An endophilin-dynamin complex promotes budding of clathrin-coated vesicles during synaptic vesicle recycling. J Cell Sci 124:133-143.

Stowell MH, Marks B, Wigge P, McMahon HT (1999) Nucleotide-dependent conformational changes in dynamin: evidence for a mechanochemical molecular spring. Nat Cell Biol 1:27-32.

Takei K, McPherson PS, Schmid SL, De Camilli P (1995) Tubular membrane invaginations coated by dynamin rings are induced by GTP-gamma $S$ in nerve terminals. Nature 374:186-190.

Takei K, Mundigl O, Daniell L, De Camilli P (1996) The synaptic vesicle cycle: a single vesicle budding step involving clathrin and dynamin. J Cell Biol 133:1237-1250.

Takei K, Slepnev VI, Haucke V, De Camilli P (1999) Functional partnership between amphiphysin and dynamin in clathrin-mediated endocytosis. Nat Cell Biol 1:33-39.

Taraska JW, Zagotta WN (2010) Fluorescence applications in molecular neurobiology. Neuron 66:170-189.

Vijayakrishnan N, Woodruff EA 3rd, Broadie K (2009) Rolling blackout is required for bulk endocytosis in non-neuronal cells and neuronal synapses. J Cell Sci 122:114-125.

Wei S, Xu Y, Shi H, Wong SH, Han W, Talbot K, Hong W, Ong WY (2010) EHD1 is a synaptic protein that modulates exocytosis through binding to snapin. Mol Cell Neurosci 45:418-429.

Zhang P, Hinshaw JE (2001) Three-dimensional reconstruction of dynamin in the constricted state. Nat Cell Biol 3:922-926.

Zhang Q, Li Y, Tsien RW (2009) Response to comment on "The dynamic control of kiss-and-run and vesicular reuse probed with single nanoparticles." Science 325:1499. 